\title{
Improved bounds on the number of low-degree points on certain curves
}

by

\author{
Pavlos Tzermias (Knoxville, TN)
}

1. Introduction. Let $\mathbb{Q}$ be the field of rational numbers and $\overline{\mathbb{Q}}$ a fixed algebraic closure of $\mathbb{Q}$. If $C$ is a smooth projective curve defined over $\mathbb{Q}$, a point $P \in C(\overline{\mathbb{Q}})$ is said to be of degree $k$ over $\mathbb{Q}$ if its field of definition is an extension of $\mathbb{Q}$ of degree $k$. If $C$ is a smooth plane curve of gonality $\gamma$ (i.e., $\gamma$ is the smallest degree of a morphism from $C$ to $\mathbb{P}^{1}$ ), a point on $C$ of degree at most $\gamma-1$ over $\mathbb{Q}$ is called a low-degree point on $C$. Under certain (and quite general) conditions, the set of low-degree points on such a curve $C$ is finite, as proven by Debarre and Klassen ([DK]) using results of Faltings $([\mathrm{F}])$. In what follows, we exclude any discussion of the case $k=1$ (i.e. the case of $\mathbb{Q}$-rational points). For some Fermat curves of prime degree $p \geq 5$, explicit (full or partial) results describing the low-degree points have appeared in the literature (see [GR], [KT], [T1], [T2], [T3], [MT]). For results regarding higher-degree points on certain Fermat curves, we refer the reader to $[\mathrm{S}]$. Recall that the Fermat curve $F_{p}$ of degree $p$ is given by the equation $X^{p}+Y^{p}+Z^{p}=0$. We also denote by $H_{5}$ the Hurwitz-Klein curve given by the equation $X^{4} Y+Y^{4} Z+Z^{4} X=0$; the curve $H_{5}$ is also known as the Snyder quintic. As explained in [T3], $H_{5}$ is a quotient of $F_{13}$.

The purpose of this paper is to improve the bounds obtained in [T2] and [T3] on the number of points of degree 6 on $F_{11}$, the number of points of degree 3 on $H_{5}$ and the number of points of degree 3 on $F_{13}$. Note that by [GR], [T3], all points on these curves of degree lower than the one indicated above have been explicitly determined; in each case, there are only two such points and they are quadratic over $\mathbb{Q}$. Our main tool will be the remarkable improvement of Coleman's effective Chabauty bound $([\mathrm{C}])$ given by Lorenzini and Tucker in [LT].

Identify the symmetric group $S_{3}$ with the group of automorphisms of the Fermat curve obtained by permuting the letters $X, Y$ and $Z$. Also denote by $\varrho$ the 3 -cycle in $S_{3}$ defined by $\varrho(X, Y, Z)=(Y, Z, X)$. Then $\varrho$ (viewed

2000 Mathematics Subject Classification: Primary 11G30, 14H25. 
both as an automorphism of $F_{13}$ and of $H_{5}$ ) commutes with the morphism $F_{13} \rightarrow H_{5}$ described in [T3]. The following two results improve Theorem 1.2 in [T2] and Theorem 1.2 in [T3], respectively:

ThEOREM 1.1. There exist at most 84 points of degree 6 on $F_{11}$ and the Galois orbit of each of these points equals its $S_{3}$-orbit.

THEOREM 1.2. There exist at most 21 cubic points on $H_{5}$ and at most 15 cubic points on $F_{13}$. The Galois orbit of each of these points equals its $\langle\varrho\rangle$-orbit.

The statements about the Galois orbits have already been proven in [T2] and [T3], so it remains to establish the stated bounds in the above theorems. For the reader's convenience, we recall that the bounds obtained in [T2] and [T3] gave at most $120\left(\right.$ resp. 33, 27) such points on $F_{11}$ (resp. $\left.H_{5}, F_{13}\right)$.

2. Proof of Theorem 1.1. Let $C$ be a smooth projective model of the curve obtained as the quotient of $F_{11}$ by the action of $S_{3}$. Both $C$ and the projection map $\phi: F_{11} \rightarrow C$ are defined over $\mathbb{Q}$. In [T2] we showed that $C$ has genus 5, its Jacobian has Mordell-Weil rank 1 over $\mathbb{Q}$ and the Galois orbits of points of degree at most 6 on $F_{11}$ are in bijective correspondence with the $\mathbb{Q}$-rational points on the curve $C$. Moreover, an affine model for $C$ is given by

$$
\begin{aligned}
\mathcal{E}: & r^{11}+22 r^{10}-11 r^{9} s+121 r^{9}-187 r^{8} s+44 r^{7} s^{2}-374 r^{8}-616 r^{7} s+528 r^{6} s^{2} \\
& -77 r^{5} s^{3}-4004 r^{7}+3432 r^{6} s+605 r^{5} s^{2}-550 r^{4} s^{3}+55 r^{3} s^{4}+1672 r^{6} \\
& +13332 r^{5} s-7590 r^{4} s^{2}+440 r^{3} s^{3}+154 r^{2} s^{4}-11 r s^{5}+39523 r^{5} \\
& -30481 r^{4} s-3905 r^{3} s^{2}+3597 r^{2} s^{3}-319 r s^{4}-30250 r^{4}-45331 r^{3} s \\
& +31064 r^{2} s^{2}-3652 r s^{3}-108009 r^{3}+117557 r^{2} s-20625 r s^{2} \\
& +164450 r^{2}-57453 r s-63151 r-1=0 .
\end{aligned}
$$

We will now use the Lorenzini-Tucker result ([LT]) to give a new upper bound on the number of $\mathbb{Q}$-rational points on $C$. The argument is very similar to the one given in [T2], but we include it here for the sake of completeness. Note that $F_{11}$ has good reduction at $p=5$, hence so does $C$. Let $\widetilde{C}$ denote a smooth projective model of the reduction of $C$ at $p=5$. Applying Theorem 1.1 of [LT] (where $p=5$ and $d=2$ ) gives

$$
\# C(\mathbb{Q}) \leq \# \widetilde{C}\left(\mathbb{F}_{5}\right)+10 .
$$

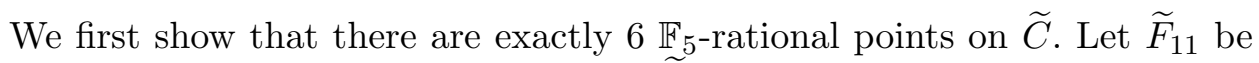
the reduction of $F_{11}$ at $p=5$. Also let $\widetilde{\mathcal{E}}$ denote the projectivization of the singular model of $\widetilde{C}$ obtained by reducing $\mathcal{E}$ at $p=5$. We have morphisms 
of curves

$$
\widetilde{F}_{11} \stackrel{\widetilde{\phi}}{\rightarrow} \widetilde{C} \stackrel{\widetilde{\pi}}{\rightarrow} \widetilde{\mathcal{E}}
$$

where $\widetilde{\pi}$ is the normalization map and $\widetilde{\phi}$ is the reduction of $\phi$ at $p=5$. Clearly, any $\mathbb{F}_{5}$-rational point on $\widetilde{C}$ maps to an $\mathbb{F}_{5}$-rational point on $\widetilde{\mathcal{E}}$ under $\tilde{\pi}$. It is straightforward to check that $\widetilde{\mathcal{E}}$ has exactly 6 points defined over $\mathbb{F}_{5}$, namely the points $(r, s)$ with coordinates $(1,0),(1,1),(1,2),(2,1)$, $(3,4)$ and the unique point at infinity. Now each of the five affine points listed above is a nonsingular point of $\widetilde{\mathcal{E}}$, so its fiber under $\tilde{\pi}$ consists of a unique $\mathbb{F}_{5}$-rational point on $\widetilde{C}$. The point at infinity on $\widetilde{\mathcal{E}}$ is singular. We claim that, among the points in its fiber under $\tilde{\pi}$, there is exactly one which is defined over $\mathbb{F}_{5}$.

To see this, note that any such point $P$ lifts under $\widetilde{\phi}$ to a point at infinity $R$ (i.e. one of the projective coordinates of $R$ vanishes). Since $P$ is $\mathbb{F}_{5}$-rational, every Galois conjugate of $R$ belongs to the fiber $\widetilde{\phi}^{-1}(P)$, which in turn consists of the $S_{3}$-conjugates of $R$. If $R$ is not defined over $\mathbb{F}_{5}$, then it is of degree 5 over $\mathbb{F}_{5}$, because the cyclotomic polynomial of degree 10 splits into a product of two irreducible factors of degree 5 over $\mathbb{F}_{5}$. Since there can be at most two $S_{3}$-conjugates of $R$ with the same coordinate vanishing, we have a contradiction. It follows that $R$ has to be equal to $(0,-1,1),(-1,0,1)$ or $(-1,1,0)$, and this proves that there exists exactly one such point $P$.

Therefore, there are exactly $6 \mathbb{F}_{5}$-rational points on $\widetilde{C}$. This implies that there are at most $6+10=16 \mathbb{Q}$-rational points on $C$. Now the three $\mathbb{Q}$ rational and the two quadratic points on $F_{11}$ project to two distinct $\mathbb{Q}$ rational points on $C$ under the morphism $\phi$. Therefore, there are at most 14 $\mathbb{Q}$-rational points on $C$ which lift to points of degree 6 on $F_{11}$. Therefore, there are at most $14 \cdot 6=84$ points of degree 6 on $F_{11}$. This completes the proof of Theorem 1.1.

It should be noted that there are at least 6 known points of degree 6 on $F_{11}$; these points are obtained by intersecting $F_{11}$ with the line $X+Y+$ $Z=0$ in $\mathbb{P}^{2}$. An easy calculation shows that these points are of the form $(c,-1-c, 1)$, where $c$ is a root of the equation

$$
X^{6}+3 X^{5}+7 X^{4}+9 X^{3}+7 X^{2}+3 X+1=0 .
$$

Note also that the action of $S_{3}$ on $F_{11}$ permutes the above points.

3. Proof of Theorem 1.2. Let $X$ denote a smooth projective model of the curve obtained as the quotient of $H_{5}$ by the action of $\langle\varrho\rangle$. Both $X$ and the natural projection map $\Phi: H_{5} \rightarrow X$ of degree 3 are defined over $\mathbb{Q}$. The genus of $X$ equals 2. As shown in [T3], the Jacobian of $X$ has Mordell-Weil rank 1 over $\mathbb{Q}$ and the Galois orbits of points of degree 1 or 3 on $H_{5}$ are in bijective correspondence with the $\mathbb{Q}$-rational points on $X$. Note that the 
two quadratic points on $H_{5}$ are fixed by $\varrho$, so their images under $\Phi$ are not $\mathbb{Q}$-rational.

We now produce an explicit model for $X$ :

Proposition 3.1. An affine model for $X$ is given by

$$
\mathcal{X}: r^{4}-4 s r^{2}-3 s r+4 r+s^{3}+2 s^{2}+s+3=0 .
$$

Proof. Let $h(r, s)$ be the left-hand side of the above equation. Consider the rational map

$$
\ominus: \mathbb{C}^{2} \rightarrow \mathbb{C}^{2}
$$

given by $(x, y) \mapsto(r, s)$, where

$$
r=x+\frac{1}{y}+\frac{y}{x}, \quad s=y+\frac{1}{x}+\frac{x}{y} .
$$

Let $\mathcal{H}_{5}$ be the affine curve $x^{4} y+y^{4}+x=0$. It suffices to show that $\ominus$ induces, by restriction, a rational map $\psi: \mathcal{H}_{5} \rightarrow \mathcal{X}$ whose fiber above $(r, s)$ equals

$$
\left\{(x, y),\left(\frac{1}{y}, \frac{x}{y}\right),\left(\frac{y}{x}, \frac{1}{x}\right)\right\}
$$

for all but finitely many $(r, s) \in \mathcal{X}(\mathbb{C})$. First we compute the fibers of $\ominus$. Fix $(r, s) \in \mathbb{C}^{2}$ and $(x, y) \in \ominus^{-1}(r, s)$. We claim that

$$
\ominus^{-1}(r, s)=\left\{(x, y),\left(\frac{1}{y}, \frac{x}{y}\right),\left(\frac{y}{x}, \frac{1}{x}\right),\left(\frac{1}{y}, \frac{1}{x}\right),\left(\frac{y}{x}, y\right),\left(x, \frac{x}{y}\right)\right\} .
$$

It is clear that all of the above six points are in $\ominus^{-1}(r, s)$. Now note that for any $(c, d) \in \ominus^{-1}(r, s)$, we have

$$
d^{3}-s d^{2}+r d-1=0, \quad d c^{2}+(1-r d) c+d^{2}=0 .
$$

Therefore, there are at most six possible values for the pair $(c, d)$ and this proves the claim. Now a straightforward calculation shows that

$$
h(\ominus(x, y))=\frac{\left(x^{4} y+y^{4}+x\right)\left(x^{4} y^{3}+y^{4}+x^{3}\right)}{x^{4} y^{4}} .
$$

In particular, $\psi$ is a rational map from $\mathcal{H}_{5}$ to $\mathcal{X}$ and for $(r, s) \in \mathcal{X}(\mathbb{C})$ it follows that, for each $(x, y) \in \ominus^{-1}(r, s)$, either $(x, y)$ or $(1 / y, 1 / x)$ is on $\mathcal{H}_{5}$. Note that, with the exception of finitely many cases, only one of the latter two points can lie on $\mathcal{H}_{5}$. By the above calculation of the fibers of $\ominus$ and the evident symmetry of $\psi$, the assertion follows.

Now we are ready to prove Theorem 1.2. Note that $F_{13}$ has good reduction at $p=5$, hence so do $H_{5}$ and $X$. Let $\widetilde{X}$ denote a smooth projective model of the reduction of $X$ at $p=5$. Applying Theorem 1.1 of [LT] (where $p=5$ and $d=1$ ) gives

$$
\# X(\mathbb{Q}) \leq \# \widetilde{X}\left(\mathbb{F}_{5}\right)+2
$$


We first show that there are exactly $6 \mathbb{F}_{5}$-rational points on $\widetilde{X}$. Let $\widetilde{H}_{5}$ be the reduction of $H_{5}$ at $p=5$. Also let $\widetilde{\mathcal{X}}$ denote the projectivization of the singular model of $\widetilde{X}$ obtained by reducing $\mathcal{X}$ at $p=5$. We have morphisms of curves

$$
\widetilde{H}_{5} \stackrel{\widetilde{\Phi}}{\rightarrow} \widetilde{X} \stackrel{\widetilde{\Pi}}{\rightarrow} \widetilde{\mathcal{X}}
$$

where $\widetilde{\Pi}$ is the normalization map and $\widetilde{\Phi}$ is the reduction of $\Phi$ at $p=5$. Clearly, any $\mathbb{F}_{5}$-rational point on $\tilde{X}$ maps to an $\mathbb{F}_{5}$-rational point on $\tilde{\mathcal{X}}$ under $\widetilde{\Pi}$. It is straighforward to check that $\widetilde{\mathcal{X}}$ has exactly 7 points defined over $\mathbb{F}_{5}$, namely the points $(r, s)$ with coordinates $(1,1),(1,3),(1,4),(3,1),(4,3)$, $(4,0)$, and the unique point at infinity. Now the point at infinity and each of the first five affine points listed above is a nonsingular point on $\widetilde{\mathcal{X}}$, so its fiber under $\widetilde{I}$ consists of a unique $\mathbb{F}_{5}$-rational point on $\widetilde{X}$. The point $(4,0)$ on $\widetilde{\mathcal{X}}$ is singular. We claim that none of the points in its fiber under $\widetilde{\Pi}$ is defined over $\mathbb{F}_{5}$.

Suppose that this is not the case. Let $P$ be an $\mathbb{F}_{5}$-rational point on $\widetilde{X}$ such that $\widetilde{\Pi}(P)=(4,0)$. Let $R$ be a point on $\widetilde{H}_{5}$ such that $\widetilde{\Phi}(R)=P$. Note that $R$ has coordinates $(c, d)$ such that

$$
d^{3}+4 d-1=0, \quad c d^{2}+d+c^{2}=0, \quad c^{3}-4 c^{2}-1=0 .
$$

Now, over $\mathbb{F}_{5}$, we have the factorizations $d^{3}+4 d-1=(d-2)\left(d^{2}+2 d+3\right)$ and $c^{3}-4 c^{2}-1=(c-3)\left(c^{2}-c+2\right)$. Note that we cannot have $(c, d)=(3,2)$, because then $c d^{2}+d+c^{2} \neq 0$. So we are left with three cases to consider:

CASE $1: d=2$ and $c \neq 3$. Since $P$ is $\mathbb{F}_{5}$-rational, the Galois conjugate $R^{\sigma}=(2 / c, 2)$ of $R$ satisfies $\widetilde{\Phi}\left(R^{\sigma}\right)=P$. In other words, $R^{\sigma}$ is a $\langle\varrho\rangle$-conjugate of $R$, so it equals either $(1 / 2, c / 2)$ or $(2 / c, 1 / c)$. Since $c \notin \mathbb{F}_{5}$, we get a contradiction.

CASE 2: $d \neq 2$ and $c=3$. As in the previous case, the Galois conjugate $R^{\sigma}=(3,3 / d)$ equals either $(1 / d, 3 / d)$ or $(d / 3,1 / 3)$. Since $d \notin \mathbb{F}_{5}$, we get a contradiction.

CASE 3: $d \neq 2$ and $c \neq 3$. Note that $3 d+1$ is a root of the polynomial $T^{2}-T+2$, therefore, $c=3 d+1$ or $c=-3 d$. In the former case, we have $R^{\sigma}=(1-1 / d, 3 / d)$ and, as before, $R^{\sigma}$ must equal either $(1 / d, 3+1 / d)$ or $(d /(3 d+1), 1 /(3 d+1))$, a contradiction, since $d \notin \mathbb{F}_{5}$. In the latter case, $R^{\sigma}=(1 / d, 3 / d)$ and, as before, it must equal either $(1 / d,-3)$ or $(-1 / 3,-1 / 3 d)$, a contradiction, since $d \notin \mathbb{F}_{5}$. This proves the claim.

Therefore, there are exactly $6 \mathbb{F}_{5}$-rational points on $\widetilde{X}$, so there are at most $6+2=8 \mathbb{Q}$-rational points on $X$. One of these points is the projection of a $\mathbb{Q}$-rational point on $H_{5}$, so it must be discarded. Therefore there are at most $7 \mathbb{Q}$-rational points on $X$ which lift to cubic points on $H_{5}$, so there are at most 21 cubic points on $H_{5}$, and this is our upper bound. As explained 
in [T3], the six known cubic points on $H_{5}$ (obtained by intersecting $H_{5}$ with the line $X+Y+Z=0$ or the conic $X Y+Y Z+Z X=0)$ do not lift to cubic points on $F_{13}$. Hence, there are at most 15 cubic points on $F_{13}$ and this completes the proof.

Acknowledgments. I thank Dino Lorenzini for encouraging me to use the effective Chabauty bounds given in [LT] in the context of [T2] and [T3] and for his comments on this work. I also thank the referee for his/her suggestions on a previous version of this manuscript.

\section{References}

[C] R. Coleman, Effective Chabauty, Duke Math. J. 52 (1985), 765-770.

[DK] O. Debarre and M. Klassen, Points of low degree on smooth plane curves, J. Reine Angew. Math. 446 (1994), 81-87.

[F] G. Faltings, Diophantine approximation on abelian varieties, Ann. of Math. 133 (1991), 549-576.

[GR] B. Gross and D. Rohrlich, Some results on the Mordell-Weil group of the Jacobian of the Fermat curve, Invent. Math. 44 (1978), 201-224.

[KT] M. Klassen and P. Tzermias, Algebraic points of low degree on the Fermat quintic, Acta Arith. 82 (1997), 393-401.

[LT] D. Lorenzini and T. Tucker, Thue equations and the method of Chabauty-Coleman, Invent. Math. 148 (2002), 47-77.

[MT] W. McCallum and P. Tzermias, On Shafarevich-Tate groups and the arithmetic of Fermat curves, in: London Math. Soc. Lecture Note Ser. 303 (special volume in honor of P. Swinnerton-Dyer), Cambridge Univ. Press, 2003, 203-226.

[S] O. Sall, Points algébriques de petit degré sur les courbes de Fermat, C. R. Acad. Sci. Paris Sér. I. Math. 330 (2000), 67-70.

[T1] P. Tzermias, Algebraic points of low degree on the Fermat curve of degree seven, Manuscripta Math. 97 (1998), 483-488.

[T2] —, Parametrization of low-degree points on a Fermat curve, Acta Arith. 108 (2003), 25-35.

[T3] —, Low degree points on Hurwitz-Klein curves, Trans. Amer. Math. Soc. 356 (2004), 939-951.

Department of Mathematics

University of Tennessee

Knoxville, TN 37996-1300, U.S.A.

E-mail: tzermias@math.utk.edu

Received on 30.8.2004

and in revised form on 22.11.2004 